\title{
A SIMPLIFIED NEUROSURGICAL TECHNIQUE FOR APPROACHING AND DAMAGING THE REGION OF THE GLOBUS PALLIDUS IN PARKINSON'S DISEASE*
}

\author{
BY
}

\section{S. OBRADOR}

From the Department of Neurosurgery, Instituto de Investigaciones Clinicas y Médicas, Madrid

Since Victor Horsley first attempted the treatment of involuntary movements by removing motor cortex, many operations have been tried for tremor and other related symptoms. Recently the interest of the neurosurgeons has been focused on the region of the globus pallidus and its pathways for the production and maintenance of Parkinsonian symptoms (Meyers, 1942; Cooper, 1953; Spiegel and Wycis, 1954). The great advantage claimed for this type of subcortical operation is that tremor and rigidity are improved or relieved without affecting voluntary power.

The operations on the pallidal region can be divided into three main groups: A, Stereotaxic approach and production of lesions by electrolysis or coagulation (Spiegel and Wycis, 1954; Hassler and Riechert, 1954); B, open approach to the subcortical regions (Meyers, 1942; Guiot and Brion, 1953); C, closed methods of tapping, coagulating, or chemical destruction (Fénelon, 1950; Cooper, 1954; Cooper and Poloukhine, 1955).

The stereotaxic methods are undoubtedly the most exact, but also slow, time-consuming, and tedious for a busy neurosurgical department. On the other hand, the open methods of approach involve a definite surgical risk, and this has to be considered carefully in the treatment of diseases that have no spontaneous mortality.

For all these reasons, we have tried to develop a quick, safe, and surgically closed method for reaching and placing lesions in the pallidal region of Parkinsonian patients, and we have evolved a technique which we have used in six cases. The relatively large size of this region makes the closed approach easy and fairly accurate. Although we cannot speak about the long-term results of this operation, as sufficient time has not yet elapsed, we have however considered it worth reporting our * Communication given at a meeting of the Society of British surgical methods for making small and deep cerebral lesions.

\section{Technique}

We shall describe very briefly our procedure, which may be separated into the following stages:-

(1) Marking the site for a trephine hole on the temporal region: this is at the intersection of two lines, one drawn $1 \mathrm{~cm}$. anterior to the intermeatal plane, and the other parallel to the upper margin of the zygoma and $3.5 \mathrm{~cm}$. above it (Fig. 1). If the pineal region is calcified, a previous lateral radiograph is useful in estimating this

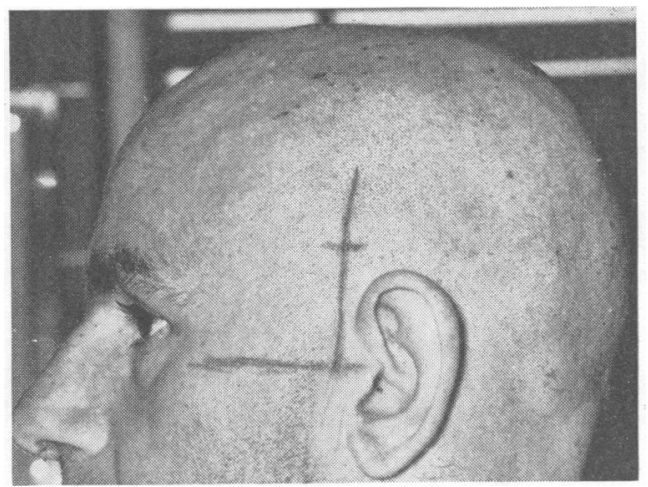

Fig. 1.-Surface marking for temporal burr-hole. See text for measurements.

point. A small amount of dye is injected on the periosteum for marking the area of the burr-hole.

(2) Under local anaesthesia a small burr-hole is made at this site, and the distance from the cortex to the skin is measured. Galea and skin are sutured.

(3) In the $x$-ray room adjacent to the operating theatre a pneumoencephalogram is carried out by cisternal puncture.

(4) Our special cannula (Fig. 2) is inserted to a depth of $4.5 \mathrm{~cm}$. from the cortex plus the distance from the cortex to the skin which was previously measured. The direction of the cannula is kept parallel to the intermeatal plane 


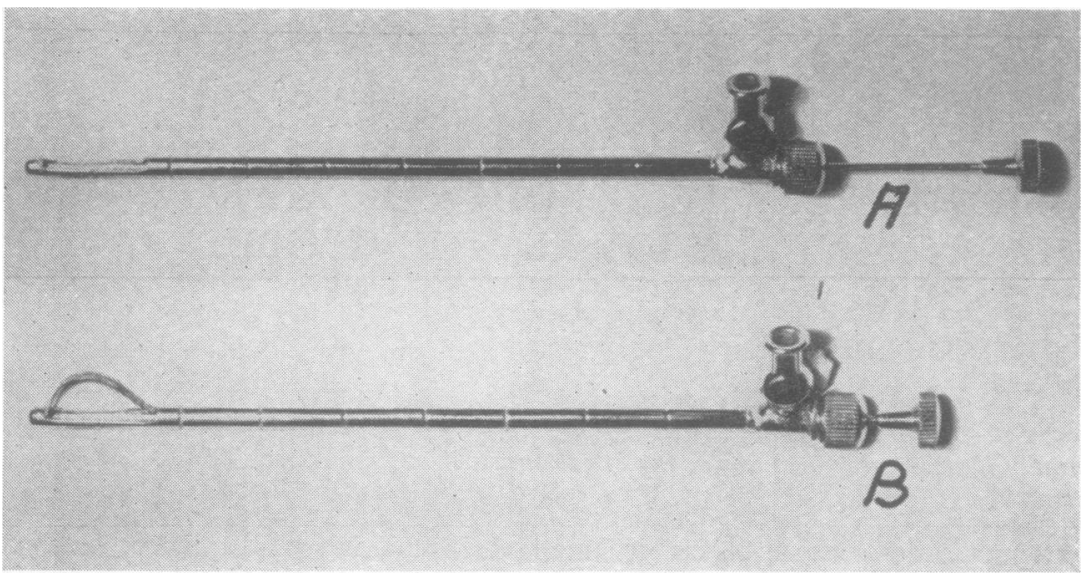

Fig. 2.-Special cannula: $A$ inserted; $B$ with leucotom wire protruding.

and is inserted horizontally or with a slight downward inclination, as seen in Fig. 3.

(5) The special cannula is $9 \mathrm{~cm}$. long and can be used as an electrode electrically insulated up to within $2 \mathrm{~mm}$. of the tip, also for injecting chemical substances, and finally as a damaging instrument by pushing inside a wire that protrudes about $10 \mathrm{~mm}$. at the side end of the cannula (Fig. 2). This last principle is similar to the primitive leucotome of Egas Moniz and Almeida Lima, and the protrusion of the wire can also be graduated from 5 to $10 \mathrm{~mm}$. The lateral opening of the instrument is known from the outside because it corresponds with the side-fitting for making the injections. The important point is to combine in a single cannula the possibilities of stimulating deeply at its end, injecting chemical substances, and making deep and small mechanical lesions.

(6) The position of the cannula is checked by radiograph film in the antero-posterior position (Fig. 3). A metal marker, $1 \mathrm{~cm}$. long, is placed on the scalp for measurement purposes. The tip of the instrument should

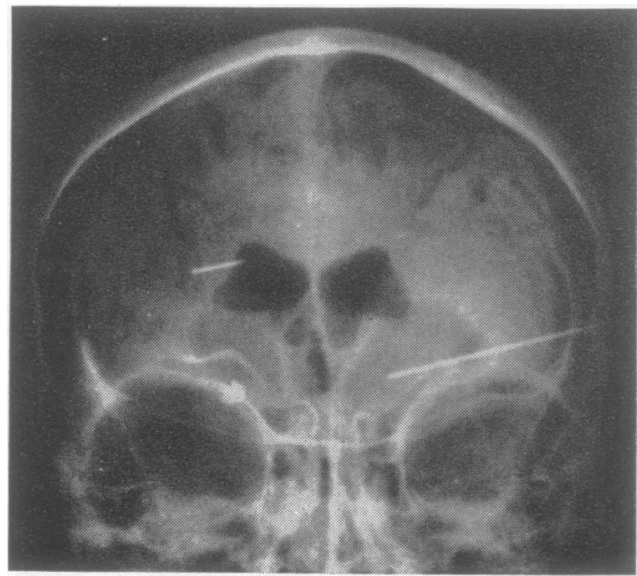

FIG. 3.-Position of cannula and metal marker after cisternal encephalogram. be about 13 to $15 \mathrm{~mm}$. from the midline (third ventricleb and corresponding to the pallidal region in the planes $25 \mathrm{~mm}$. anterior and $6 \mathrm{~mm}$. below the pineal, accordin to the reference of Spiegel and Wycis (1952) in the book (Fig. 4). The tip of the cannula in this positiof reaches the AB line of Cooper and Poloukhine (1955).

(7) Once the position is estimated to be correct ㅁํ음

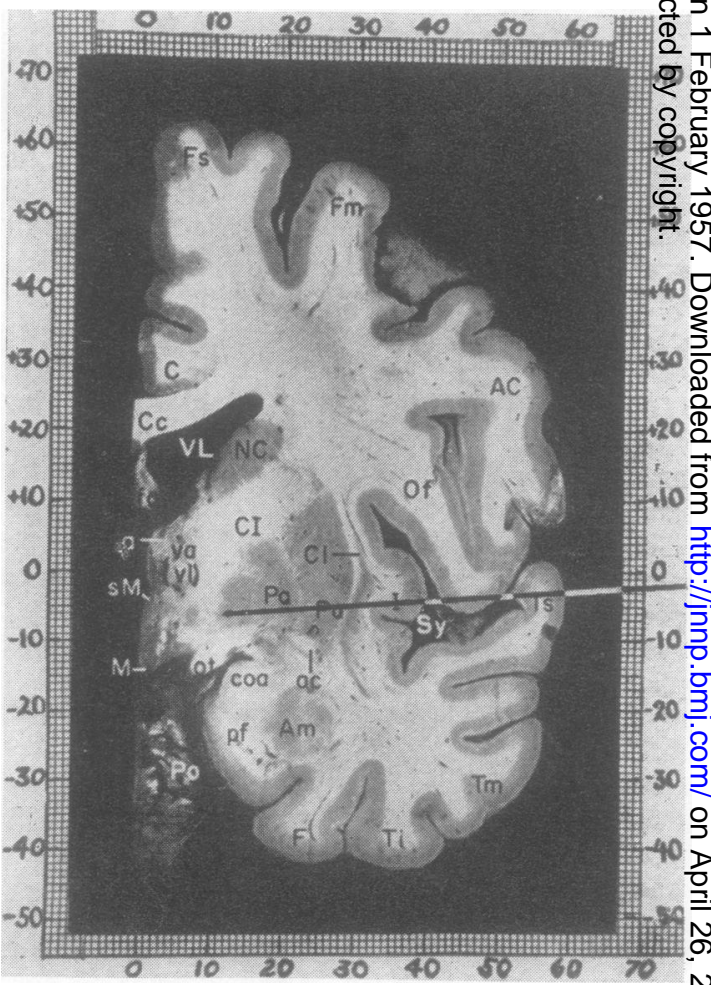

FIG. 4.-Coronal section of brain showing path of cannula and site of lesion in the globus pallidus. 
monopolar stimulation is carried out to see that there are no contralateral motor responses from the internal capsule.

(8) A small amount of $2 \%$ procaine solution ( $0 \cdot 25-$ $0.5 \mathrm{ml}$.) is injected, and the effect on the tremor and rigidity is observed. If the cannula is in the pallidal region, these symptoms disappear at once. An electromyogram can be recorded during this stage.

(9) The cutting wire of the cannula is pushed out and rotated several times to damage the region of the globus pallidus. Smaller lesions can be made by partial rotation.

The whole procedure takes only about half an hour.

\section{Complications and Immediate Results}

Of the six patients operated on, two had clear evidence of bilateral Parkinsonism, and in four the affection was predominantly one-sided. Altogether, 10 pallidal lesions have been made: two bilateral lesions and two repeated on the same side. The ages of the patients varied from 37 years to 58 years.

The most frequent complication immediately after operation has been motor weakness in the contralateral limbs: this was observed after five of the 10 operations. In one case, spastic weakness of the arm has persisted, but in the others the weakness cleared up within a few hours or days.

In one case there was a transient disturbance of motor speech lasting for two weeks. In another, there was contralateral hypoaesthesia for nine days. A persisting contralateral homonymous quadrantic field defect occurred in another case.

In the case of bilateral operations, it appears that the interval between the two operations should not be too short. In our first patient, who had bilateral operations with an interval of eight days, we observed after the second operation a curious and alarming incapacity for facial movements, phonation, and swallowing which last for several weeks. With this experience in mind, we deferred the second operation in the second case of bilateral lesions for two months. In this patient the only complication was a tendency to adduction in the gait, and some difficulty in the automatism of walking for nine days.

There is no doubt from our observations that a pallidal lesion produced in the manner described above can effect an immediate abolition or great diminution of the contralateral rigidity and tremor of the Parkinsonian patient, without inducing paralysis. In some cases rigidity and tremor may recur after a few days or weeks, but usually with less intensity than before operation. Our follow-up extends only over a period of a few months, but all the patients seem to have improved to some extent, and none has been made worse except the case cited of persisting pyramidal damage producing spastic weakness of the left upper limb instead of the preoperative rigidity and tremor. Of the other five patients, three have maintained their improvement, and in two initial improvement has been less well maintained. In general, rigidity seems to benefit more than tremor, and in the case of bilateral lesions one side may benefit more than the other.

Further experience and a longer follow-up are obviously necessary to evaluate the therapeutic results of these pallidal operations. An important point which we have in mind and hope to settle is the advisability of repeated unilateral or bilateral lesions in those cases in which symptoms recur after initial improvement.

\section{Summary}

A simplified and quick neurosurgical procedure is described for inserting a specially designed cannula into the region of the globus pallidus through the temporal route in Parkinsonian patients.

This special cannula can be used as a deep stimulating electrode to test that the tip is not in the internal capsule; as a needle for seeing the action on the tremor and rigidity after injecting small amounts of procaine solution; and also as a cutting instrument for placing deep lesions in the pallidal region.

The position of the cannula is checked radiographically by means of cisternal pneumoencephalography.

The immediate results in the first six patients are briefly reported.

\section{REFERENCES}

Cooper, I. S. (1953). Science, 118, 193.

(1954). Ibid., 119, 417.

- , - and Poloukhine, N. (1955). J. Amer. Geriat. Soc., 3, 839

Fénelon, F. (1950). Rev. neurol. (Paris), 83, 437.

Guiot, G., and Brion, S. (1953). Ibid., 89, 578.

Hassler, R., and Riechert, T. (1954). Nervenarzt, 25, 441.

Meyers, R. (1942). Res. Publ. Ass. nerv. ment. Dis., $21,602$.

Spiegel, E. A., and Wycis, H. T. (1952). Stereoencephalotomy. Grune and Stratton, New York. 